94

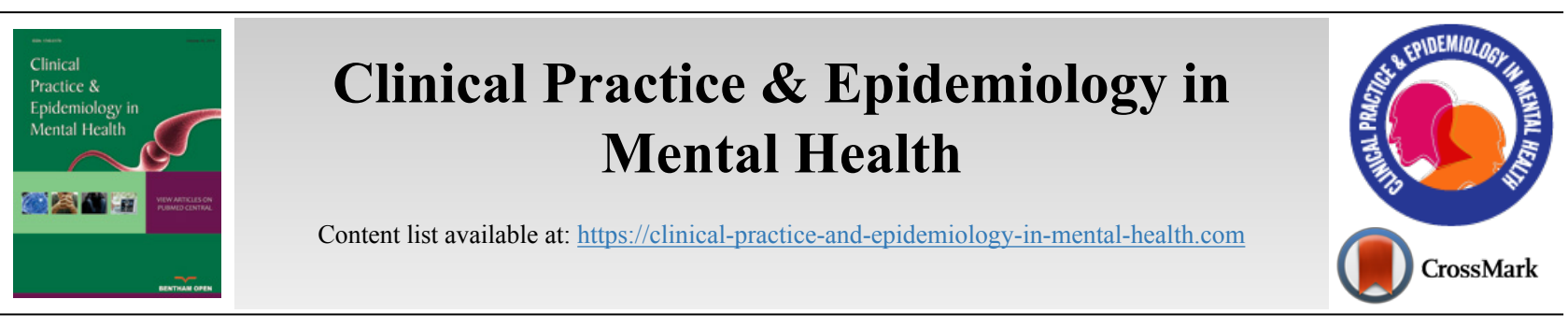

$\mathbf{E}^{\prime}, \mathbf{T} 25, \$ /$

\title{
Autistic Traits and Illness Trajectories
}

\author{
Liliana Dell'Osso ${ }^{1}$, Primo Lorenzi ${ }^{1}$ and Barbara Carpita ${ }^{1, *}$ \\ ${ }^{\prime}$ Department of Clinical and Experimental Medicine, University of Pisa, Pisa, Italy
}

\begin{abstract}
In the framework of increasing attention towards autism-related conditions, a growing number of studies have recently investigated the prevalence and features of sub-threshold Autistic Traits (ATs) among adults. ATs span across the general population, being more pronounced in several clinical groups of patients affected by psychiatric disorders. Moreover, ATs seem to be associated with specific personality features in nonclinical population, implying both a higher vulnerability towards psychopathology and extraordinary talents in specific fields. In this framework, the DSM-5's Autism Spectrum Disorder (ASD) presentations may be considered as the tip of an iceberg that features several possible clinical and non-clinical phenotypes. Globally, the autism spectrum may be considered as a trans-nosographic dimension, which may not only represent the starting point for the development of different psychopathological trajectories but also underlie non-psychopathological personality traits. These different trajectories might be shaped by the specific localization and severity of the neurodevelopmental alteration and by its interaction with the environment and lifetime events. In this wider framework, autistic-like neurodevelopmental alterations may be considered as a general vulnerability factor for different kinds of psychiatric disorders, but also the neurobiological basis for the development of extraordinary abilities, eventually underlying the concept of geniality. Moreover, according to recent literature, we hypothesize that ATs may also be involved in the functioning of human mind, featuring the peculiar sense of "otherness" which can be found, with different grades of intensity, in every human being.
\end{abstract}

In the last decades, an increasing number of studies stressed on the hypothesis that a series of alterations in the development of the "social brain" might represent the neurobiological underpinning of several different clinical conditions that require clinical attention in both childhood and adult life [1 6]. A growing number of data suggest that these neurodevelopmental alterations might be considered as the neurostructural correlates of the peculiar perception of "otherness" and "diversity" typical of the human mind, as well as of a variety of human behaviors, the origin of which generated speculations since the earliest recorded history [7]. From a psychopathological point of view, an altered neurodevelopmental pathway (particularly, not exclusively, concerning the social brain) may be associated with an "autistic" spectrum [5]. The label "autism" is employed in this case due to the presence of some psychopathological features in these subjects which, in their extreme presentation, resemble those observed for the first time in patients affected by the clinical and more severe phenotypes of autism spectrum disorders. In this framework, the clinical diagnosis of autism spectrum disorder (ASD) may be conceptualized as the end of

\footnotetext{
* Address correspondence to this author at the Department of Clinical and Experimental Medicine, University of Pisa, Italy;

E-mail: liliana.dellosso@med.unipi.it
}

the Gaussian distribution of the autism spectrum, with a clinical threshold distinguishing the general population from the clinical population [8 - 11].

ASD is a new diagnostic category proposed in the fifth edition of the Diagnostic and Statistical Manual of Mental Disorders (DSM-5), which identifies patients with severe impairment in social communication and social interaction, together with the presence of restrictive and repetitive behaviors, interests or activities. ASD replaces a set of diagnoses that were included in DSM-IV, and particularly in Autistic Disorder, Pervasive Developmental Disorder Not Otherwise Specified (PDDNOS) and Asperger's Disorder. These diagnoses were previously grouped in a chapter labeled "Pervasive Developmental Disorders". To date, epidemiological studies on ASD report a prevalence in 1 out of 68 children (1:42 boys; 1:189 girls) [12]. DSM-5's ASD may be better considered as an "enlarged category" in which the relevance of "intellectual impairment" has been erased to encompass the Asperger's Syndrome and other milder but clinical, full-threshold presentations of the same quality, differing only in symptomatological severity [10, 13]. The classification proposed in DSM-5 also features neurodevelopmental disorders other than ASD, such as attention-deficit hyperactivity disorder, intellectual disabilities, communication disorder, specific learning disorder, motor 
disorders [14]. These clinical presentations are deeply intertwined and widely overlap with ASD, suggesting the presence of a continuum which encompasses the broad variety of ASD phenotypes, including different kinds of clinical expressions of neurodevelopmental alterations.

Moreover, DSM-5 does not take into account the broader spectrum of sub-threshold manifestations distributed in a continuum in a non-clinical population, as well as the broad presence of autistic-like symptoms in clinical population of patients affected by other psychiatric conditions $[10,13]$. Subthreshold "Autistic Traits" (ATs) (milder in severity but of the same quality as the clinical manifestations of ASD) have been firstly highlighted by studies conducted among unaffected firstdegree relatives of probands with ASD $[15,9]$. Subsequently, they were demonstrated to be variously distributed in general population and in particular in some high-risk groups [16 - 18], such as university students (and particularly scientists) [19 22]. Intriguingly, in the latter group a high prevalence of social anxiety has also been reported, a condition frequently associated with both substance abuse and ATs, which is supposed to mask autism spectrum symptoms among females $[20,23,24,25]$. ATs also seem highly prevalent in a broad variety of clinical groups (e.g., among patients with eating disorders, mood disorders, borderline personality disorder), where they might represent a specific risk factor for suicide ideation and behaviors [26 - 32]. Considering the increasing number of studies which stress on the relevance of ATs in nonASD clinical samples [33, 26 - 32], it has been suggested that different kinds of psychiatric conditions may be developed as a consequence of a neurodevelopmental alteration similar to the one linked to ASD; the specific localization and severity of this neurodevelopmental alteration, together with the interaction with the environment during lifetime, may lead to different psychopathological trajectories [5, 34, 35].

In this framework, the DSM-5's description of ASD may be considered as the tip of an iceberg which features several possible clinical and non-clinical phenotypes. In particular, recent literature highlighted the relevance, for a better understanding of psychopathology, of investigating the prevalence and features of ATs in clinical and non-clinical populations, which may not only represent the starting point for the development of different kinds of psychiatric disorders, but also underlie non-psychopathological personality characteristics $[12,22,33]$. The manifestation of ATs may change during the lifetime, with several presentations overlapping each other. When they do not evolve into full-blown clinical symptoms of any kind, they still generally imply the presence of slight autistic-like deficits, particularly in social communication, featuring an area of vulnerability open to several trajectories with a significant impact on the quality of life (including impairments in relationships and reciprocal communication skills) leading to poorer outcomes in education, employment and community inclusion. However, in some cases, development towards hyper-adaptive behaviors is also possible, eventually allowing these subjects, when these traits meet favorable environmental conditions, to live highly successful lives [7, 13, 36].

Globally, as suggested by other authors, we may conceptualize the broad spectrum of these specific features, with a possible shared neurobiological basis, as a "trait", a dimension (the core of which can be associated with autistic-like features) continuously distributed from general to clinical population, rather than as manifestations of different (full-threshold or subthreshold) conditions [37, 38]. As reported above, the dimensional conceptualization proposed here is based on a wide body of evidence from literature and can be summarized in the following points:

- Sub-threshold ATs are continuously distributed in the general population; their expression may vary depending on their quantity and quality as well as on the interaction with the environment;

- Among full-threshold manifestations, the ASD phenotypes are the tip of the iceberg of several possible clinical expressions (e.g., schizophrenia, bipolar disorder, eating disorders, borderline personality disorder) underlying the autism spectrum. This fact is well reflected in the widespread comorbidities of ASD, as well as in the high prevalence of ATs in clinical samples $[33,13,22]$;

- Sub-threshold ATs may represent the underlying correlates of several subclinical or isolated symptoms, personality characteristics, and temperamental traits, representing a vulnerability factor for the development of psychiatric disorders;

- Finally, as we will discuss further below, ATs may be involved in the functioning of mind, featuring the peculiar sense of "otherness" and "diversity" which can be partially found, with different grades of intensity, in every human being, but which is also a special "trademark" of autistic mind. In this wider framework, ATs may be considered as a general vulnerability factor for developing symptoms after life events, but also as one of the premises for the development of extraordinary talents in specific fields of interest, possibly underlying the concept of geniality $[7,13,36]$.

The reported high rates of comorbidity among different mental disorders (as described by the categorical approach of DSM) suggest that the current diagnostic categories may share more similarities than differences [2 - 5]. This evidence raised increasing interest in the development of a more comprehensive framework, which would be able to feature cognitive, affective, and neurobiological processes shared by several psychiatric syndromes [34, 2 - 4]. Given the high amount of evidence about the possible role of the autism spectrum in the development of different psychiatric disorders [33, 26 - 32], as well as about its measurable neurobiological correlates $[9,39,40]$ the autism spectrum model possibly seems to be a useful tool for this purpose.

As stated in a study [13], the autism spectrum constitute both its clinical and subclinical dimensions: it is a heterogeneous construct and its core features are variously expressed across the population with many potentially different outcomes. The autism spectrum model features a dimensional and quantitative understanding of autism spectrum as it can improve our 
capability to build a theoretical framework for the reassessment of data extracted from neurobiological, psychopathological, clinical and epidemiological studies [13]. It is a transnosographic concept that may allow rethinking of all psychiatric disorders from a broader point of view, possibly leading towards a neuro-psychogenetic approach which might overcome the categorical and descriptive approach of contemporary psychopathology.

However, there is also another point of interest linked to this model. Understanding ATs and their neurobiological underpinnings may shed more light on the neurobiological source of human diversity. According to this hypothesis, ATs may be the basis of atypical behaviors linked to independent thinking and originality, as well as of subjective feelings of individuality and otherness. These personality traits constitute both a possible vulnerability factor concerning life events and a possible hyper-adaptive element for specific fields of interest and activities. ATs may be the underpinning of the cognitive restructuration and problem-solving processes of those individuals who do not share the vision of their group, but are a mixture of special skills and fragilities [7, 13]. On the other hand, the intertwined relationship between autism spectrum, traumatic events and mood symptoms might be of particular interest in order to clarify the role of life events in influencing illness trajectories in psychopathology [41 - 43].

In this framework, it is also possible that the autism spectrum model would allow reaching a new point of view about some of the long-standing questions about human nature, in particular, it might allow clarifying the neurobiological basis of creativity and geniality, as far as the divergent thinking is concerned in our conceptualization of these features. Moreover, at the same time, it might shed more light on the pathogenesis of psychopath mind, considering the frequently highlighted strong link between genius and psychopaths. Creativity seems to follow a linear distribution in the general population like other features, such as blood pressure or height. Creativity is a rare feature placed at the far end of a continuously distributed variability [7]. Although ASD has been considered as a condition which implies difficulties in abstract thinking, with a lack of creativity, when investigating creativity amongst subjects of the autism spectrum some researchers highlighted a dramatically higher prevalence than in the general population, leading to rethinking of this concept $[44,7]$. Despite that, it is noteworthy that in the sample on which this study was conducted there were subjects with a lower IQ and also with full-blown intellectual disability. Globally, it seems that the autism spectrum should be considered as an area where extraordinary abilities meet the most severe deficits, featuring both the extreme ends of the human brain functioning $[44,7]$. This is the area of so-called "Savant Syndrome" classically known with the French name, Idiots Savants. However, there is another question worth investigating: why most of the subjects with ASD, or even ATs do not develop savant skills [7]?

As reported by Snyder [45], this issue is probably related to the reported possibility of acquiring new skills after neurological damage, or after being exposed to repetitive transcranial magnetic stimulation (rTMS). Snyder's controversial theory identifies the top-down inhibition as the mechanism which ultimately prevents us from being creative. People having autism spectrum due to the different structure of their brain could be more frequently able to bypass top-down inhibition as well as neurotypical subjects when their temporal lobe is reversibly disabled with rTMS. Avoiding top-down inhibition may allow gaining privileged access to raw forms of information not available otherwise, leading to a perceptual insight more adherent to reality in contrast with expectationbiased interpretations. It is also possible that all the subjects with ASD or ATs may have the potential to develop savant skills, the outcome of which would be determined by the interaction between their specific neurodevelopmental trajectory and different kinds of environmental factors [45, 7].

In the last decades, several questionnaires have been designed to assess ATs in the general population, such as the Social Communication Questionnaire (SCQ) [46], the Broader Autism Phenotype Questionnaire (BAPQ) [47], the Social Responsiveness Scale (SRS) [48], the Autism-Spectrum Quotient (AQ) [19] and the Adult Autism Sub-threshold Spectrum (AdAS spectrum) [18]. While these instruments have opened broader perspectives for researchers in this field, the main challenge for the future is to identify the specific neurobiological asset (including all the possible morphological, neurophysiological, biochemical, metabolic phenotypes) associated with ATs, as well as to clarify the possible pathways that may lead to different psychopathological (and non-psychopathological) conditions. In a further perspective, a better understanding of this matter may also shed light on the very structure of the human mind.

\section{REFERENCES}

[1] Brüne M, Ribbert H, Schiefenhövel W. The Social Brain: Evolution and Pathology. Chichester: Wiley 2003.

[http://dx.doi.org/10.1002/0470867221]

[2] Peled A. Brain "Globalopathies" cause mental disorders. Med Hypotheses 2013; 81(6): 1046-55.

[http://dx.doi.org/10.1016/j.mehy.2013.09.032] [PMID: 24161400]

[3] Doherty JL, Owen MJ. Genomic insights into the overlap between psychiatric disorders: Implications for research and clinical practice. Genome Med 2014; 6(4): 29.

[http://dx.doi.org/10.1186/gm546] [PMID: 24944580]

[4] Peled A, Geva AB. "Clinical brain profiling": a neuroscientific diagnostic approach for mental disorders. Med Hypotheses 2014; 83(4): 450-64.

[http://dx.doi.org/10.1016/j.mehy.2014.07.013] [PMID: 25155246]

[5] Dell'Osso L, Dalle Luche R, Maj M. Adult autism spectrum as a transnosographic dimension. CNS Spectr 2016; 21(2): 131-3. [http://dx.doi.org/10.1017/S1092852915000450] [PMID: 26349624]

[6] Porcelli S, Van Der Wee N, van der Werff S, et al. Social brain, social dysfunction and social withdrawal. Neurosci Biobehav Rev 2019; 97: 10-33.

[http://dx.doi.org/10.1016/j.neubiorev.2018.09.012] [PMID: 30244 163]

[7] Happé F, Frith U. The beautiful otherness of the autistic mind. Philos Trans R Soc Lond B Biol Sci 2009; 364(1522): 1346-50. [http://dx.doi.org/10.1098/rstb.2009.0009] [PMID: 19528016]

[8] Grove R, Baillie A, Allison C, Baron-Cohen S, Hoekstra RA. Empathizing, systemizing, and autistic traits: Latent structure in individuals with autism, their parents, and general population controls. J Abnorm Psychol 2013; 122(2): 600-9.

[http://dx.doi.org/10.1037/a0031919] [PMID: 23713510]

[9] Billeci L, Calderoni S, Conti E, et al. The Broad Autism (Endo)Phenotype: Neurostructural and Neurofunctional Correlates in Parents of Individuals with Autism Spectrum Disorders. Front Neurosci 2016; 10: 346. 
[http://dx.doi.org/10.3389/fnins.2016.00346] [PMID: 27499732]

[10] Dell'Osso L, Luche RD, Gesi C, Moroni I, Carmassi C, Maj M. From Asperger's Autistischen Psychopathen to DSM-5 Autism Spectrum Disorder and Beyond: A Subthreshold Autism Spectrum Model. Clin Pract Epidemiol Ment Health 2016; 12: 120-31.

[http://dx.doi.org/10.2174/1745017901612010120] [PMID: 27867417]

[11] Dell'Osso L, Lorenzi P. L'ombra dell'autismo Declinazioni cliniche e psicopatologiche dello Spettro Autistico Sottosoglia. Milano: FrancoAngeli 2018.

[12] Centers for Disease Control and Prevention (CDCP). Community report from the autism and developmental disabilities monitoring (ADDM). Atlanta, GA: network. national center on birth defects and developmental disabilities 2014.

[13] Dell'Osso L, Muti D, Carpita B, et al. The Adult Autism Subthreshold Spectrum (AdAS) model: A neurodevelopmental approach to mental disorders. Journal of Psychopathology 2018; 24: 118-24. a

[14] American Psychiatric Association. Diagnostic and statistical manual of mental disorders. 5th ed. Washington, DC: Author 2013.

[15] Losh M, Adolphs R, Poe MD, et al. Neuropsychological profile of autism and the broad autism phenotype. Arch Gen Psychiatry 2009; 66(5): 518-26

[http://dx.doi.org/10.1001/archgenpsychiatry.2009.34] [PMID: 19414711]

[16] Skylark WJ, Baron-Cohen S. Initial evidence that non-clinical autistic traits are associated with lower income. Mol Autism 2017; 8: 61 [http://dx.doi.org/10.1186/s13229-017-0179-z] [PMID: 29158888]

[17] Suzuki T, Miyaki K, Eguchi H, Tsutsumi A. Distribution of autistic traits and their association with sociodemographic characteristics in Japanese workers. Autism 2018; 22(8): 907-14 [http://dx.doi.org/10.1177/1362361317716605] [PMID: 28901163]

[18] Dell'Osso L, Gesi C, Massimetti E, et al. Adult Autism Subthreshold Spectrum (AdAS Spectrum): Validation of a questionnaire investigating subthreshold autism spectrum. Compr Psychiatry 2017; 73: 61-83.

[http://dx.doi.org/10.1016/j.comppsych.2016.11.001] [PMID: 27918948]

[19] Baron-Cohen S, Wheelwright S, Skinner R, Martin J, Clubley E. The autism-spectrum quotient (AQ): Evidence from Asperger syndrome/high-functioning autism, males and females, scientists and mathematicians. J Autism Dev Disord 2001; 31(1): 5-17. [http://dx.doi.org/10.1023/A:1005653411471] [PMID: 11439754]

[20] Jobe LE, White SW. Loneliness, social relationships, and a broader autism phenotype in college students. Pers Individ Dif 2007; 42: 1479-89.

[http://dx.doi.org/10.1016/j.paid.2006.10.021]

[21] Trevisan D, Birmingham E. Examining the relationship between autistic traits and college adjustment. Autism 2016; 20(6): 719-29. [http://dx.doi.org/10.1177/1362361315604530] [PMID: 26471426]

[22] Dell'Osso L, Carpita B, Cremone IM, et al. The mediating effect of trauma and stressor related symptoms and ruminations on the relationship between autistic traits and mood spectrum 2018. [http://dx.doi.org/10.1016/j.psychres.2018.10.040]

[23] Sbrana A, Bizzarri JV, Rucci P, et al. The spectrum of substance use in mood and anxiety disorders. Compr Psychiatry 2005; 46(1): 6-13. [http://dx.doi.org/10.1016/j.comppsych.2004.07.017] [PMID: 15714188]

[24] Attwood T, Grandin T, Faherty C, et al. Asperger's and Girls. Arlington, TX: Future Horizons 2006

[25] Marazziti D, Abelli M, Baroni S, et al. Recent findings on the pathophysiology of social anxiety disorder. Clin Neuropsychiatry 2014; 11: 91-100.

[26] Kato K, Mikami K, Akama F, et al. Clinical features of suicide attempts in adults with autism spectrum disorders. Gen Hosp Psychiatry 2013; 35(1): 50-3.

[http://dx.doi.org/10.1016/j.genhosppsych.2012.09.006] [PMID: 23141028]

[27] Takara K, Kondo T. Comorbid atypical autistic traits as a potential risk factor for suicide attempts among adult depressed patients: A casecontrol study. Ann Gen Psychiatry 2014; 13(1): 33. [http://dx.doi.org/10.1186/s12991-014-0033-z] [PMID: 25328535]

[28] Dell'Osso L, Conversano C, Corsi M, et al. Polysubstance and behavioral addictions in a patient with bipolar disorder: Role of lifetime subthreshold autism spectrum. Case Rep Psychiatry 2018; 20181547975

$$
\text { [http://dx.doi.org/10.1155/2018/1547975] [PMID: 29682383] }
$$

[29] Dell'Osso L, Cremone IM, Carpita B, et al. Correlates of autistic traits among patients with borderline personality disorder. Compr Psychiatry
2018; 83: 7-11.

[http://dx.doi.org/10.1016/j.comppsych.2018.01.002]

[PMID: 29500962

[30] Dell'Osso L, Carpita B, Gesi C, et al. Subthreshold autism spectrum disorder in patients with eating disorders. Compr Psychiatry 2018; 81: 66-72.

[http://dx.doi.org/10.1016/j.comppsych.2017.11.007] [PMID: 29268154]

[31] Dell'Osso L, Carpita B, Muti D, et al. Mood symptoms and suicidality across the autism spectrum. Compr Psychiatry 2019; 91: 34-8. [http://dx.doi.org/10.1016/j.comppsych.2019.03.004]

[PMID: 31003723

[32] Dell'Osso L, Bertelloni CA, Di Paolo M, et al. Problematic internet use in university students attending three superior graduate schools in italy: Is autism spectrum related to suicide risk? Int J Environ Res Public Health 2019; 16(7)E1098

[http://dx.doi.org/10.3390/ijerph16071098] [PMID: 30934756]

[33] Anckarsäter H, Stahlberg O, Larson T, et al. The impact of ADHD and autism spectrum disorders on temperament, character, and personality development. Am J Psychiatry 2006; 163(7): 1239-44.

[http://dx.doi.org/10.1176/ajp.2006.163.7.1239] [PMID: 16816230]

[34] White PD, Rickards H, Zeman AZ. Time to end the distinction between mental and neurological illnesses. BMJ 2012; 344e3454 [http://dx.doi.org/10.1136/bmj.e3454] [PMID: 22628005]

[35] Ruzich E, Allison C, Smith P, et al. Measuring autistic traits in the general population: A systematic review of the Autism-Spectrum Quotient (AQ) in a nonclinical population sample of 6,900 typical adult males and females. Mol Autism 2015; 6: 2 .

[http://dx.doi.org/10.1186/2040-2392-6-2] [PMID: 25874074]

[36] Dell'Osso L, Lorenzi P. Genio e follia 20 Il complepsso rapporto tra spettro autistico e competenze eccezionali. Milano: FrancoAngeli 2019.

[37] Constantino JN, Todd RD. Autistic traits in the general population: A twin study. Arch Gen Psychiatry 2003; 60(5): 524-30. [http://dx.doi.org/10.1001/archpsyc.60.5.524] [PMID: 12742874]

[38] Constantino JN, Charman T. Diagnosis of autism spectrum disorder: reconciling the syndrome, its diverse origins, and variation in expression. Lancet Neurol 2016; 15(3): 279-91.

[http://dx.doi.org/10.1016/S1474-4422(15)00151-9] [PMID: 26497771]

[39] Carpita B, Muti D, Dell'Osso L. Oxidative stress, maternal diabetes, and autism spectrum disorders. Oxid Med Cell Longev 2018; 20183717215

[http://dx.doi.org/10.1155/2018/3717215] [PMID: 30524654]

[40] Carpita B, Marazziti D, Palego L, et al. Microbiota, immune system and autism spectrum disorders. an integrative model towards novel treatment options. Curr Med Chem 2019; •.. Epub ahead of print [http://dx.doi.org/10.2174/0929867326666190328151539]

[41] Dell'Osso L, Dalle Luche R, Carmassi CA. New perspective in posttraumatic stress disorder: Which role for unrecognized autism spectrum? Int J Emerg Ment Health 2015; 17: 436-8.

42] Dell'Osso L, Corsi M, Gesi C, et al. Adult Autism Subthreshold Spectrum (AdAS Spectrum) in parents of pediatric patients with epilepsy: Correlations with post-traumatic stress symptoms. Compr Psychiatry 2018; 83: 25-30.

[http://dx.doi.org/10.1016/j.comppsych.2018.02.004] [PMID: 29549876]

[43] Carmassi C, Corsi M, Bertelloni CA, et al. Mothers and fathers of children with epilepsy: Gender differences in post-traumatic stress symptoms and correlations with mood spectrum symptoms. Neuropsychiatr Dis Treat 2018; 14: 1371-9. [http://dx.doi.org/10.2147/NDT.S158249] [PMID: 29872304]

[44] Howlin P, Goode S, Hutton J, Rutter M. Savant skills in autism: Psychometric approaches and parental reports. Philos Trans R Soc Lond B Biol Sci 2009; 364(1522): 1359-67. [http://dx.doi.org/10.1098/rstb.2008.0328] [PMID: 19528018]

[45] Snyder A. Explaining and inducing savant skills: Privileged access to lower level, less-processed information. Philos Trans R Soc Lond B Biol Sci 2009; 364(1522): 1399-405.

[http://dx.doi.org/10.1098/rstb.2008.0290] [PMID: 19528023]

[46] Berument SK, Rutter M, Lord C, Pickles A, Bailey A. Autism screening questionnaire: Diagnostic validity. Br J Psychiatry 1999; 175: 444-51.

[http://dx.doi.org/10.1192/bjp.175.5.444] [PMID: 10789276]

[47] Hurley RS, Losh M, Parlier M, Reznick JS, Piven J. The broad autism phenotype questionnaire. J Autism Dev Disord 2007; 37(9): 1679-90. [http://dx.doi.org/10.1007/s10803-006-0299-3] [PMID: 17146701] 
[48] Constantino JN, Davis SA, Todd RD, et al. Validation of a brief quantitative measure of autistic traits: Comparison of the social responsiveness scale with the autism diagnostic interview-revised. J Autism Dev Disord 2003; 33(4): 427-33.

[http://dx.doi.org/10.1023/A:1025014929212] [PMID: 12959421]

\section{(C) 2019 Dell'Osso et al.}

This is an open access article distributed under the terms of the Creative Commons Attribution 4.0 International Public License (CC-BY 4.0), a copy of which is available at: (https://creativecommons.org/licenses/by/4.0/legalcode). This license permits unrestricted use, distribution, and reproduction in any medium, provided the original author and source are credited. 